\title{
Towards a Digital Twin Framework for Adaptive Last Mile City Logistics
}

\author{
Abdelhadi Belfadel \\ Institute for Technological Research \\ IRT SystemX \\ Lyon, France \\ abdelhadi.belfadel@irt-systemx.fr
}

\author{
Jakob Puchinger \\ Institute for Technological Research \\ IRT SystemX \\ Palaiseau, France \\ jakob.puchinger@irt-systemx.fr
}

\author{
Sebastian Hörl \\ Institute for Technological Research \\ IRT SystemX \\ Palaiseau, France \\ sebastian.horl@irt-systemx.fr
}

\author{
Rodrigo Javier Tapia \\ Delft University of Technology \\ Delft, Netherlands \\ R.J.Tapia@tudelft.nl
}

\begin{abstract}
The use of real-time data in logistics is an important topic. Every day, logistics produces a large quantity of data, which is mainly produced by monitoring and controlling the enormous flow of goods. The last-mile delivery market is expanding at a rapid pace through large- and small-scale consumer platforms, but the economic drivers to create more sustainable systems are weak. Therefore, cities are facing the potential downside of this "Uberisation" of logistics. Urban and city planners, city administrators, and business stakeholders need a new adaptive approach such as the usage of digital twinning solutions of urban logistics. This to help in the interpretation of the dynamics of logistics networks in the city and the consequences of the introduction of particular innovations. The challenge is to predict the most likely developments for the coming years and propose feasible policies on that basis. This research work aims to advance research in the field of digital twins applied to city logistics, by proposing a framework enabling new applications for designing and assessing targeted urban logistics policies and to develop a range of logistics solutions for shared, connected, and low-emission logistics operations, empowered by an adaptive modeling approach.
\end{abstract}

Index Terms - digital twin, last mile delivery, urban logistics

\section{INTRODUCTION}

The surge of on-demand logistics is placing a significant strain on last-mile delivery systems [1]. Consumer preferences require responsive logistics systems that provide customized products at a low cost. The last-mile delivery market is expanding at a rapid pace through large- and small-scale consumer platforms, but the economic drivers to create more sustainable systems are weak [2]. Therefore, cities are facing the potential downside of this "Uberisation" of logistics.

There is uncertainty about how the actual business will evolve and what direction will be taken by the leading companies (such as development of large warehouses near cities, the introduction of very small warehouses in neighbourhoods or the development of the physical internet and asset sharing [3]). Hence, the most likely outcomes for the coming years

\footnotetext{
Corresponding author: Abdelhadi Belfadel (abdelhadi.belfadel@irtsystemx.fr)
}

cannot be reliably anticipated and feasible policies cannot be proposed. Urban and city planners, city administrators, and business stakeholders need new adaptive approaches such as digital twinning solutions of urban logistics to support experimentation, simulation and decision-making for on-demand logistics operations. The goal is to help in the interpretation of the dynamics of logistics networks in the city and the consequences of the introduction of particular innovations.

The idea is to anticipate the possible consequences of market-led developments, assessing rapid-response green approaches, and introducing progressive changes by adopting novel business models that foster public-private partnerships. To do this, models driven by data should work in parallel with real experiments to replicate results and predict outcomes of response actions. In recent years, cities are already evolving in this direction with a rising number of logistics living labs, which complement the old "predict and provide" paradigm [4]. However, applications of digital twin frameworks can be found mainly in the context of product management, shop floor and production management [5], [6], and the problem we are facing is that it has become clear that existing architectures are too generic for usage in logistics [6].

Digital twins can play an important role in supporting experimentation in the planning and development of urban logistics policies. However, research in the area of digital twins applied to urban freight planning is still in its infancy [4]. Moreover, urban freight planning has longer feedback loops that depend on the interaction of multiple independent agents, which adds to the complexity of the twinning. The present paper contributes to research in the field of digital twins applied to city logistics by proposing a framework for designing and assessing targeted urban logistics policies to develop a range of logistics solutions for shared, connected, and low-emission logistics operations, empowered by an adaptive modeling approach.

To reach this objective, this paper is organized as follows: Section II focuses on the related work. Afterward, Section 
III focuses on the principal building blocks of the proposed framework by introducing each building block and its objective. Section IV presents the ongoing implementation of the proposed framework, along with a description of the targeted application scenario in section V. Finally, conclusions and future work are covered in Section VII.

\section{RELATED WORK}

The continuous development in sensor technologies, IoT, and big data analytics has led to advancements in digital twins that enable connecting the physical and digital world [7]. Logistics objects are equipped with comprehensive sensor systems, and a digital image of the respective logistics objects is created, leading to a digital twin [8]. Yet, a digital twin is more than a digital representation of a physical asset. A digital twin is a comprehensive physical and functional representation of an asset that provides all the information necessary to process it throughout its life cycle [9].

However, the exact definition of a digital twin depends on the integration level. A distinction must be made between a digital model, a digital shadow and a digital twin. The core of the consideration is in all cases a physical and a digital object. In a digital model, there is only a manual data flow between the physical and the digital object. A change in the physical object has no effect on the digital object and vice versa. In a digital shadow, there is an automatic data flow in at least one direction, whereby the change of the physical object leads to a change of the digital object. However, this does not apply in the other direction. In a digital twin, data flow between the two objects is automatic. Thus, a change to the physical object leads directly to a change to the digital object and vice versa [10].

There are many contributions in the literature in this context of internet of things, big data and digital twins applied in logistics. [6] propose a digital twin architecture based on a generic architecture, which enables the analysis and processing of large amounts of data in real-time. [11] propose a solution for transportation planning of bus rapid transportation systems. The authors prototype a smart sensor to collect information from the users of the transportation system which allows to track origin and destination locations. The aim is to help administrators make the transportation planning work more efficient and reliable. [12] propose an IoT-based solution for people using public transportation. This application enables the user of public transportation to get recommendations about the route and the incidents that would happen or happened on their routes, based on previous data and its analytics. [13] introduce a system architecture that combines digital twins of logistics systems with simulation logic to start a simulation based on the current situation of the system in real-time. Real-time events produced by scanners or sensors are transmitted to the digital twin directly or via existing systems such as warehouse management systems allowing prediction of the future of the system. Those predictions assist the planner and controller in generating feasible decision scenarios based on the digital twin. In the context of logistics, [14] propose a simulationbased decision support tool for in-house logistics that analyses the activities that can occur in both a distribution facility and a production facility towards logistics 4.0. As a result, the developed simulation models can be applied in different inhouse logistics settings and function as digital twinning tools to operations improvement without disruptions in reality. [15] propose a technical framework on which a real use case of big data and IoT technologies is developed in the logistics industry. This use case combines truck telematics and geoinformation to enable proactive alerts about possible upcoming hazards which are sent to drivers. The authors have also adopted camera-based technologies for fatigue management to improve driver safety and to capture evidence of important driving events. Nevertheless, it is evident that concrete use cases for the implementation of such architectures are rarely found in logistics nor in last mile city logistics, although the implementation of digital twinning in logistics offers considerable additional value [15].

\section{PRoposed FrAMEWORK}

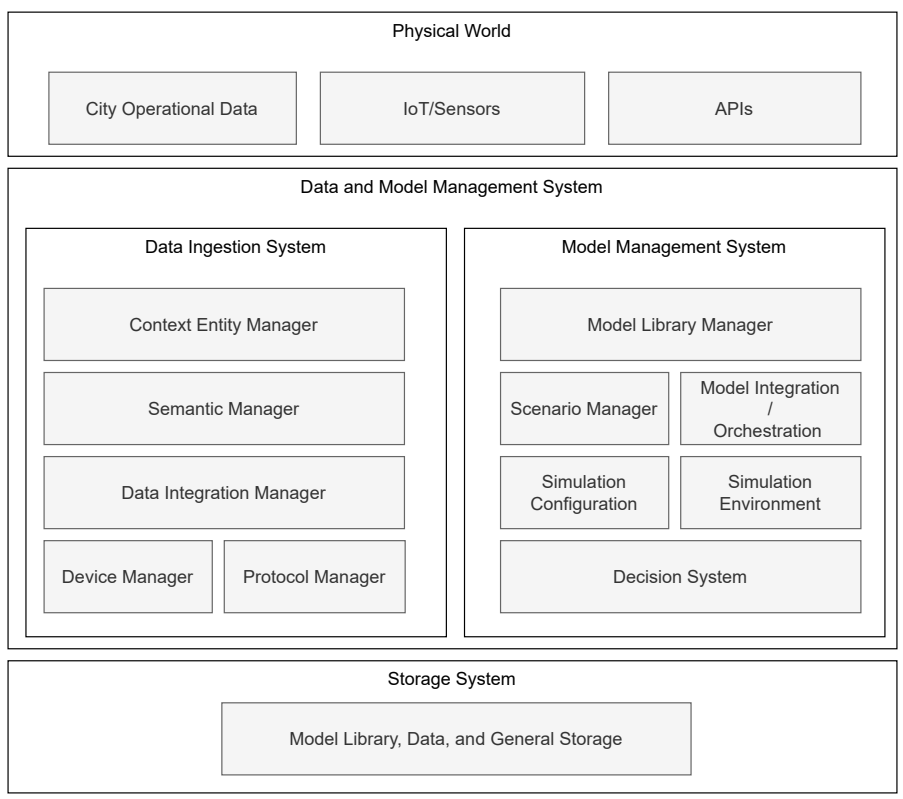

Fig. 1. Proposed Framework

The proposed Framework is depicted in Figure 1 and is composed of three main levels. The top level (Physical World) represents the external entities and sensors such as City Operational Data, IoT entities and sensors, and exposed APIs. This level is linked to the intermediate level (Data and Model Management System) that is composed of two main systems: Data Ingestion System (DIS) and Model Management System (MMS).

The DIS aims to integrate contextual entities to the system and to keep the digital twins updated, by managing the bidirectional connections with related devices that expose those contextual data through the Device and Protocol Manager 
sub-components. This enables to manage the communication protocols with the physical counterpart and exposes those contextual data to the model management system. As a digital twin requires a detailed model of its environment and physical counterpart, the DIS proposed in our framework offers a possibility to enrich the system and its artifacts with descriptions based on standard semantic annotations or ontologies (see, e.g., [16] for an ontology of city logistics) through the Semantic Manager sub-component. This allows the use of specific business-oriented semantic models to provide an integrated and detailed view of all relevant artifacts.

The third main component of the proposed Framework is the Model Management System (MMS) that manages the models library which is a set of open-source or case-specific software applications (such as agent based models, demand models or network models for route choice and assignment of delivery trips) that provides flexibility to the policies and actions that can be analysed. The MMS is composed of several sub-components including the Model Library Manager which holds and overview of all available models, their specifications and the relationship between the models and the key performance indicators (KPIs). The Scenario Manager component enables the registration of simulation scenarios, their linked models and configuration of models (contextual data, model parameters, targeted KPIs). The Model Integration and Orchestration sub-component aims to integrate and orchestrate the models with by linking data sets and other models' outputs based on the requested simulation scenarios. The Simulation Configuration sub-component aims to manage the configuration of the simulation environment from a technical point of view (virtual machine, libraries needed, etc.). Regarding the Simulation Environment sub-component, it is a run-time virtual environment generated based on the configuration of the simulation. This sub-component aims to use containerization, and virtualization to dynamically create an execution environment for each executed scenario, and, finally, to execute the targeted simulation and retrieve the outputs.

Digital twins enable real-time communication between assets and different systems. With regards to logistics, data collection alone does not represent a major challenge. The decisive factor is how this data is processed further in order to offer real added value. In this context, the added value is created with the help of KPIs tailored precisely to the targeted application. For this, the last sub-component of the MMS is the Decision System that aims to decide which results of the executed simulation scenario are most likely achievable. It examines the defined scenario KPIs and then recommends the interventions required in the physical world through the DIS to achieve the predicted outcome. This decision system builds its knowledge based on different inputs (model and scenario parameters, model outputs, predicted results, and realworld contextual data). The last level concerns the storage system for the management of model libraries, managed data, and general storage such as the simulation scenarios, their configuration, related models and data, or an ontology store with a SPARQL [17] (for SPARQL Protocol and RDF Query Language) endpoint related to the usage of the semantic manager sub-component.

The following section provides an overview of current implementation work and technical choices for implementing the components of the proposed framework, as well as a targeted application scenario.

\section{FRAMEWORK IMPLEMENTATION AND TECHNICAL CHOICES}

First steps towards the implementation of the framework have been taken. We follow an enterprise architecture development method from TOGAF [18] to analyse and identify the building blocks of the proposed framework presented in section III which captures the architecture requirements to guide the development. Each component is evaluated from several perspectives, namely the business, data, application, and technology requirements, the fundamental functionality and attributes (behaviour, interfaces provided, including security aspects), interoperability (where applicable) and dependence between building blocks. As an early output of this architecture work, we aim to use the following technologies for the implementation of each component.

For the Storage System, it will be based on several existing open source technologies and must cover a very diverse storage need. Different storage will be considered, for instance, as sensor data can be generated very fast, a traditional database can reach its limits on processing speed and size quite fast which demands a different approach for the management of this kind of data. In this case, big data technologies are proposed to get the necessary speed and scalability. Time series database like InfluxDB ${ }^{1}$, or document-oriented database such as MongoDB ${ }^{2}$ will be used for the storage and querying of the data.

Regarding the DIS, some existing open source solutions are relevant and respond to the requirements and objectives of the proposed framework. For example, the Context Entity Manager, Device, Protocol and Data Integration Manager sub-components might be implemented with Fiware Orion Context Broker ${ }^{3}$. Indeed, this solution enables to manage the entire life-cycle of contextual information including updates, queries, registrations and subscriptions, all of which are tasks that the sub-components are meant to cover. However, this solution might only be connected to devices using REST protocols through the NGSI v2 API ${ }^{4}$. This is sufficient for our targeted application scenario since we are dealing with external APIs implemented with REST (Representational State Transfer) protocol. However, in case we need a specific protocol to connect to an external sensor or device, a specific implementation of an API to interoperate with this sensor has to be implemented. Regarding the Semantic Manager, a well known triple store has been selected which is Apache Jena

\footnotetext{
${ }^{1}$ https://www.influxdata.com/

${ }^{2}$ https://www.mongodb.com/

${ }^{3}$ https://fiware-orion.readthedocs.io/en/master/

${ }^{4}$ https://fiware-ges.github.io/orion/api/v2/stable/
} 
Fuseki [19]. This latter serves RDF (Resource Description Framework) data over HTTP, and exposes a SPARQL server that provides REST-style SPARQL HTTP Update, SPARQL Query, and SPARQL Update using the SPARQL protocol over HTTP in case a semantic model is needed when integrating or sharing contextual data. For the MMS, a from scratch implementation has to be developed since there are no existing solutions to manage the model library, the scenario configuration and orchestration. However, components such as MongoDB ${ }^{5}$ will be used to expose the model library for scenario configuration and execution. Finally, we aim to use containerization and virtualization to dynamically create the simulation execution environments. These ephemeral environments will be created using the specifications provided by the model library and instantiated only when required. After execution, at each time step, these environments will be removed to conserve resources. Given the parameterization of the models and temporal requirement of execution cycles, several instances will be created dynamically for each evaluated scenario.

\section{USE CASE SCENARIO}

To give a practical view on a potential deployment of a digital twin for city logistics, an example for the city of Lyon, France, shall be presented which is currently under development in the Horizon 2020 project LEAD. The idea is to study the impact of city logistics policies on a number of high level KPIs such as congestion, noise, air pollution and GHG emissions. Specifically, the project looks at Confluence, which is an urban peninsula between the rivers Rhône and Saône. It is especially suited for experiments in urban logistics policy as it is a well-contained area, framed on two sides by rivers and in the North by rail lines (see Figure 2). Only three major road network access points to the peninsula are relevant.

In the course of the project, the idea is to assess the potential deployment of a digital twin, which is able to propose scenarios for policy change based on current and past information, can track those changes once in place, and which will be used to steer policy decisions adaptively in the future.

The underlying modeling architecture is composed by two components: MASS-GT [20], an agent-based simulation which is able to predict the decision-making, routing, and tourformation of logistics operators; and MATSim [21], an agentbased transport simulation framework which is mainly aimed at simulating the movements of people, but which into logistics agents can be integrated, which will interact with people in a detailed road network simulation. Both models are sensitive to policy decision. For instance, both the logistics operators and persons will be affected by a fee that needs to be paid by drivers when entering the area. In the case of passenger transport, agents may switch their mode of transport or avoid hours in which the policy is in place (if it is time-dependent), and logistics operators may adapt their routes to avoid the area. Another scenario to explore is the deployment of an Urban

\footnotetext{
${ }^{5}$ https://www.mongodb.com/
}

Consolidation Center (UCC) which can be used by logistics companies to perform the fine distribution of shipments in the area with low-emissions vehicles.

The system has a number of static inputs such as census data and Household Travel Survey data, which are updated annually or even less frequently to establish the demand for personal transport. A comprehensive enterprise census of France is available as open data, which is updated monthly and can be ingested via a public API. This way, it is possible to establish a baseline travel demand only based on open data [22]. Other policy-relevant data sets are available in shorter intervals such as the vehicle counts on the roads which are updated in intervals of six minutes. Furthermore, it is planned to deploy cameras at the entry points of the peninsula to perform detailed counts of commercial traffic.

The main interest in using a digital twin in the area is to relieve the population from noise and congestion and to reduce GHG emissions. In terms of policy, we can envision that the digital twin acts as a decision-support tool that helps administrators to explore various scenarios around a low- or zeroemission zone that is established on the peninsula. Possible scenarios could include the following. First, cordon pricing could be introduced for all or only commercial vehicles. In that case a weighting between different objectives becomes important: While higher prices for commercial vehicles at peak hours makes operators shift to earlier hours, the population will be affected by increasing noise levels in the morning. If commercial operations are priced out in the morning, congestion at peak times will increase. The aim of the digital twin could then be to control pricing such that a previously defined level of noise at all times of the day and a maximum level of congestion is reached. Second, the introduction of an UCC could be studied. Again, there are trade-offs to make as minimized congestion in the policy area might lead to increased traffic in the surrounding parts of the city. Again, the digital twin can make sure that policy objectives are reached (or are shown to be impossible to reach) while maintaining certain on other KPIs.

Note that by continuously ingesting congestion data from the loop detectors in the city's road network and adjusting pricing levels adaptively, a clear link is established between the politically negotiated goals and measurable effects that show that the policies put in place are efficient and effective. Such a tool, therefore, offers new ways of urban decision-making and to communicate and justify policy interventions.

\section{DISCUSSION}

As depicted in sections III and IV, the design and the technical analysis of the proposed Framework have been done by following guidelines and best practices of an Enterprise Architecture Framework (TOGAF). We identified and analyzed the architecture building blocks that capture the architecture requirements to guide the development of the required solution building blocks. Each component in the proposed Framework is analyzed considering several concepts such as the business, data, application, and technology requirements, the 


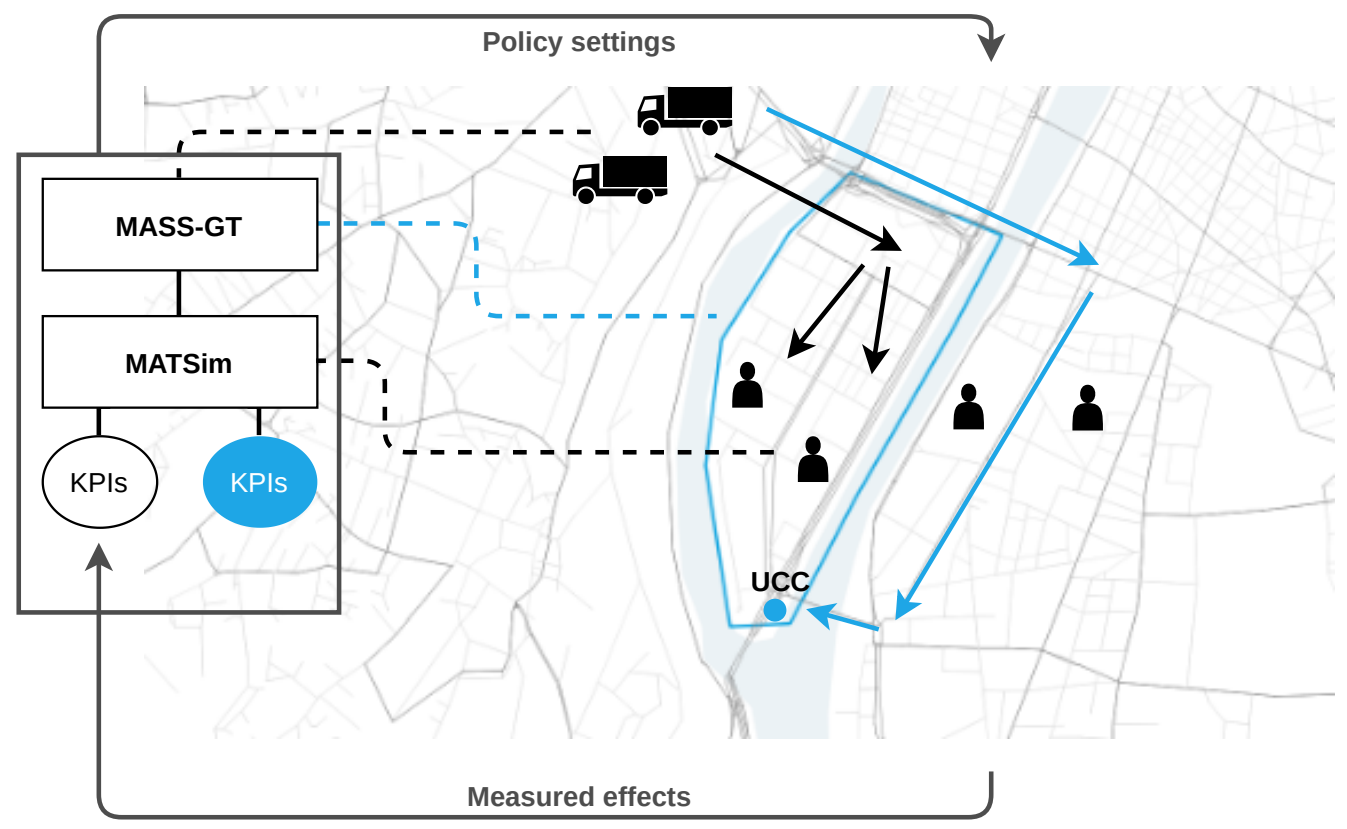

Fig. 2. Use case scenario

fundamental functionality and attributes (behavior, interfaces provided, including security aspects), interoperability (where applicable), and dependence between building blocks. This led to the referred solution building blocks that exist in the market, that are evaluated based on different criteria such as the functionality provided, parameters, or security for the implementation of the proposed Framework. The final choices have been depicted in section IV. Even though with a deeper analysis of the literature more specialized alternatives for the components could appear, the chosen solutions are complete and mature enough to meet the needs and objectives of the digital twin.

Regarding the adoption of this Framework, each simulation scenario has its requirements and, therefore, it is not necessary to use all the components of the proposed Framework. Indeed, if there is no need for an ontology in a simple scenario, there is no need, for example, to use the semantic manager.

This ongoing work developed in the scope of the project LEAD ${ }^{6}$ is under design and implementation. As future work, we aim to present in detail the architecture process and the followed methodology to select the solution building blocks, their integration, the proof of concept applied to the Lyon use case scenario as depicted in section $\mathrm{V}$, as well as the generalization of this approach for several scenarios.

\section{CONCLUSION}

To address ecological and social challenges in the modern city, fine-grained policies are necessary to respect the individual circumstances and specifics of the affected areas. To date, no comprehensive tool exist to construct, evaluate,

\footnotetext{
${ }^{6} \mathrm{https}: / /$ www.leadproject.eu/
}

implement and track pin-pointed policies in urban logistics. Yet, the technical tools such as traffic observation, finetuned measurement units and electric pricing are in place. The present paper presents a first comprehensive platform architecture to make use of such data to inform individual, data-backed and justifiable policy-making in this sector. To show that steps have been taken from the conceptual level to a concrete implementation, the use case of Lyon is presented.

With our detailed perspective on the numerous components that are necessary to define a comprehensive and sufficiently generic, and, thus, adaptable, framework we aim to foster a discussion on the architectural design of such a software package. While today, merely academic and very individualized solutions exist, it is likely that a more standardized and formalized framework will contribute to a practical implementation of such systems in cities around the world.

\section{ACKNOWLEDGMENT}

This paper presents work developed in the scope of the project LEAD. This project has received funding from the European Union's Horizon 2020 research and innovation programme under grant agreement no. 861598. The content of this paper does not reflect the official opinion of the European Union. Responsibility for the information and views expressed in this paper lies entirely with the authors.

\section{REFERENCES}

[1] M. Joerss, J. Schröder, F. Neuhaus, C. Klink, and F. Mann, "Parcel delivery. the future of last mile: Mckinsey \& company report," 2016. 
[2] J. Allen, M. Piecyk, M. Piotrowska, F. McLeod, T. Cherrett, K. Ghali, T. Nguyen, T. Bektas, O. Bates, A. Friday, S. Wise, and M. Austwick, "Understanding the impact of e-commerce on last-mile light goods vehicle activity in urban areas: The case of london," Transportation Research Part D: Transport and Environment, vol. 61, pp. 325-338, 2018. [Online]. Available: https://www.sciencedirect.com/science/article/pii/S136192091730305X

[3] ALICE-ETP, "Roadmap to The Physical Internet," ALICE-ETP, Tech. Rep., 2020. [Online]. Available: http://www.etp-logistics.eu/wp-content/uploads/2020/11/Roadmapto-Physical-Intenet-Executive-Version_Final.pdf

[4] E. Marcucci, V. Gatta, M. Le Pira, L. Hansson, and S. Bråthen, "Digital twins: A critical discussion on their potential for supporting policymaking and planning in urban logistics," Sustainability, vol. 12, no. 24 p. $10623,2020$.

[5] C. Zhuang, J. Liu, and H. Xiong, "Digital twin-based smart production management and control framework for the complex product assembly shop-floor," The International Journal of Advanced Manufacturing Technology, vol. 96, no. 1, pp. 1149-1163, 2018.

[6] H. Haße, B. Li, N. Weißenberg, J. Cirullies, and B. Otto, "Digital twin for real-time data processing in logistics," in Artificial Intelligence and Digital Transformation in Supply Chain Management: Innovative Approaches for Supply Chains. Proceedings of the Hamburg International Conference of Logistics (HICL), Vol. 27. Berlin: epubli GmbH, pp. 4-28, 2019.

[7] F. Tao, H. Zhang, A. Liu, and A. Y. Nee, "Digital twin in industry: State-of-the-art," IEEE Transactions on Industrial Informatics, vol. 15, no. 4, pp. 2405-2415, 2018.

[8] D. Wohlfeld, "Digitaler zwilling für die produktion von übermorgen," Zeitschrift für wirtschaftlichen Fabrikbetrieb, vol. 114, no. 1-2, pp. 6567, 2019.

[9] S. Boschert and R. Rosen, "Digital twin-the simulation aspect," in Mechatronic futures. Springer, pp. 59-74, 2016.

[10] W. Kritzinger, M. Karner, G. Traar, J. Henjes, and W. Sihn, "Digital twin in manufacturing: a categorical literature review and classification. ifac-papersonline 51 (11), 1016-1022 (2018)," in 16th IFAC Symposium on Information Control Problems in Manufacturing INCOM, 2018.

[11] L. F. Herrera-Quintero, K. Banse, J. Vega-Alfonso, and A. VenegasSanchez, "Smart its sensor for the transportation planning using the iot and bigdata approaches to produce its cloud services," in 2016 8th Euro American Conference on Telematics and Information Systems (EATIS). IEEE, pp. 1-7, 2016.

[12] D. Puiu, S. Bischof, B. Serbanescu, S. Nechifor, J. Parreira, and H. Schreiner, "A public transportation journey planner enabled by iot data analytics," in 2017 20th Conference on Innovations in Clouds, Internet and Networks (ICIN). IEEE, 2017.

[13] B. Korth, C. Schwede, and M. Zajac, "Simulation-ready digital twin for realtime management of logistics systems," in 2018 IEEE International Conference on Big Data (Big Data). IEEE, 2018.

[14] F. Coelho, S. Relvas, and A. Barbosa-Póvoa, "Simulation-based decision support tool for in-house logistics: the basis for a digital twin," Computers \& Industrial Engineering, vol. 153, 2021.

[15] J. Hopkins and P. Hawking, "Big data analytics and iot in logistics: a case study," The International Journal of Logistics Management, 2018.

[16] N. Anand, M. Yang, J. R. van Duin, and L. Tavasszy, "Genclon: An ontology for city logistics," Expert Systems with Applications, vol. 39, no. 15, 2012.

[17] B. DuCharme, Learning SPARQL: querying and updating with SPARQL 1.1. " O'Reilly Media, Inc.", 2013.

[18] T. O. Group, The Open Group Architecture Framework TOGAF ${ }^{\mathrm{TM}}$ Version 9. Basharat Hussain, 2009.

[19] A. Jena, "Fuseki: serving rdf data over http," 2014.

[20] M. de Bok, L. Tavasszy, and Sebastiaan Thoen, "Application of an empirical multi-agent model for urban goods transport to analyze impacts of zero emission zones in The Netherlands," Transport Policy, p. S0967070X19307383, Jul. 2020. [Online]. Available: https://linkinghub.elsevier.com/retrieve/pii/S0967070X19307383

[21] A. Horni, K. Nagel, and K. W. Axhausen, Eds., The Multi-Agent Transport Simulation MATSim. Ubiquity Press, Aug. 2016. [Online]. Available: http://www.ubiquitypress.com/site/books/10.5334/baw/

[22] S. Hörl and M. Balac, "Reproducible scenarios for agent-based transport simulation," Arbeitsberichte Verkehrs Raumplan, vol. 1499, 2020. 